\title{
Recombinant Human SCARB2 Expressed in Escherichia coli and its Potential in Enterovirus 71 Blockage
}

\author{
Hai-Vy Vo-Nguyen ${ }^{1,2} \cdot$ Thanh-Tan Nguyen ${ }^{1,2} \cdot$ Huyen-Trang Thi $\mathrm{Vu}^{3} \cdot$ Thanh-Thao Thi Nguyen $^{3} \cdot$ \\ Quoc-Cuong Hoang ${ }^{3} \cdot{\text { Thuoc Linh } \operatorname{Tran}^{1,2} \cdot \text { Hieu Tran-Van }}^{1,2}$
}

Received: 27 April 2020 / Accepted: 2 November 2020/Published online: 5 January 2021

(C) Shiraz University 2021

\begin{abstract}
Hand, foot and mouth disease is a common viral infectious disease caused by enteroviruses, including coxsackie A16 (CVA16) and enterovirus 71 (EV71). HFMD can cause severe symptoms in children which can be fatal. Human scavenger receptor class B member 2 (SCARB2) is a cellular receptor for EV71 and CVA16, providing a potential approach for preventing EV71 infection and transmission. In this present study, we constructed and assessed the potential of recombinant SCARB2, using E. coli expression system. To generate this construct, scarb2 gene was cloned into pET22b vector and expressed in E. coli BL21 (DE3). The expression of SCARB2 was induced by $0.1 \mathrm{mM}$ IPTG and analyzed using SDSPAGE, followed by Western blot. Expressed SCARB2 was in inclusion bodies and refolded to obtain the soluble form with recovery efficacy of $100 \%$. This recombinant protein was then validated for binding with EV71 via indirect ELISA in two different pHs (7.4 and 5.5), which partially revealed the mechanism of virus-receptor interaction. These results envisaged potential applications for utilizing recombinant SCARB2 in preventing the virus transmission.
\end{abstract}

Keywords EV71 $\cdot$ HFMD $\cdot$ Inclusion bodies $\cdot$ Refolding $\cdot$ SCARB2

\section{Introduction}

Hand, foot and mouth disease (HFMD) is a common viral infectious disease characterized by common symptoms including fever, painful sores in the mouth, and a rash with blisters on hands, feet and buttocks. In most cases, the disease is mild and self-limiting, occurring mainly in children under 10 years old, but it is most commonly seen in children under 5 years of age. It can also appear in adolescents and occasionally in adults. Viruses causing HFMD are spread by direct contact with saliva, mucus, fluid from blisters and stool of infected people. Adults infected with HFMD may shed the virus and have no symptoms (WHO). The dominant cause of HFMD is a

Hieu Tran-Van

tvhieu@hcmus.edu.vn

1 Faculty of Biology and Biotechnology, University of Science, Ho Chi Minh City, Vietnam

2 Vietnam National University, Ho Chi Minh City, Vietnam

3 Pasteur Institute, Ho Chi Minh City, Vietnam group of enteroviruses, including coxsackie A16 (CVA16) and enterovirus 71 (EV71) (Yamayoshi et al. 2012a). Although complications are uncommon, EV71 infection can lead to severe symptoms in children and has been associated with neurological diseases, including acute flaccid paralysis, aseptic meningitis, and brain stem encephalitis with neurogenic pulmonary edema, which can be fatal (Lee 2016). There are no specific antiviral drugs or vaccine commercially available against enteroviruses causing HFMD hitherto (WHO). EV71 belongs to the human enterovirus A species of the enterovirus genus within the family Picornaviridae (King et al. 2000). Virion includes a non-enveloped capsid surrounding a core of single-stranded, positive-polarity RNA approximately $7.5 \mathrm{~kb}$ in size (Brown and Pallansch 1995). Since its discovery in 1969, EV71 has been recognized as a frequent cause of epidemics of HFMD associated with severe neurological sequelae in a small proportion of cases (WHO 2011).

Approaches have been intensively evaluated to prevent the spread of the virus, in which vaccines are the most priority (Yi et al. 2017). Several significant milestones 
have been archived recently in vaccine development of China, which leads to its phase 3 testing on trial ( $\mathrm{Li}$ et al. 2014; Zhu et al. 2013, 2014). In December 2015 and January 2016, two HFMD vaccines approved by the China Food and Drug Administration were commercially produced and began to apply on Chinese children. However, these vaccines required long duration to demonstrate their efficacy and safety before becoming commercially available for application (Yi et al. 2017). In addition, the outreach efforts toward preventing the virus from entering its target cells also achieved certain results. Many studies were conducted to identify receptors on the surface of human cells that play a role in facilitating the infection of EV71. In particular, SCARB2 receptor presents in many cell types in the body and has been shown to be an important factor in the infection of EV71, which can be bound by all existing EV71 strains (Yamayoshi et al. 2009). Moreover, SCARB2 is also a co-receptor for CVA16, one of the main causative agents of HFMD besides EV71 (Yamayoshi et al. 2012b). SCARB2 has been confirmed to have ten potential N-glycosyl binding sites, considered to be responsible for its proper folding (Yamayoshi and Koike 2011).

Recently, recombinant receptor produced in $E$. coli has been shown to interact with EV71 in vitro (Xu et al. 2018). However, the receptor was used in insoluble form, so it is not suitable for related or further research, as well as putting into applications. On the other hand, the role of the receptor's glycosylation remained unclear in interaction with EV71. In this study, we created a SCARB2-expressing plasmid to produce recombinant receptor in $E$. coli along with a refolding procedure. Then, the interaction between recombinant SCARB2 and EV71 was evaluated by indirect ELISA.

\section{Materials and Methods}

\subsection{Bacterial Strains, Plasmids, Reagents and Growth Conditions}

The scarb2 gene was amplified from pPHAGE-C-TAPSCARB2 (Harvard PlasmID Database) (Huttlin et al. 2015). E. coli DH5 $\alpha$ and E. coli BL21 (DE3) were employed as host strains for cloning and protein expression, respectively. Both were routinely grown in LuriaBertani (LB) medium containing $100 \mu \mathrm{g} / \mathrm{mL}$ of ampicillin at $37^{\circ} \mathrm{C}$. The pET22b plasmid was used for both cloning and expression for scarb2 gene, and protein expression was controlled by T7 promoter via IPTG (isopropyl B-D-1thiogalactopyranoside) (Biobasic) inducer. DNA marker (Bioline, 33,053), protein maker (GE, 17,044,601) and prestained protein marker (Thermo Scientific, 26,612) were utilized for band size marking. Recombinant protein was constructed, expressed and stored at $-20^{\circ} \mathrm{C}$.

\subsection{Construction of Recombinant pET-SCARB2}

(Fig. 1)

pPHAGE-C-TAP-SCARB2 plasmid was used as a template for amplification of scarb2 gene by PCR with specific primers (scarb2F: CATATGatcgagaagaaaattgtg and scarb2R: CTCGAGaatcatagacttcagtcgac). scarb2 gene and pET22b plasmid were digested with NdeI and XhoI (Thermo Scientific). The ligation reaction containing the double-digested insert and expression vector was performed in the presence of T4 DNA ligase (Thermo Scientific), and the resulting mixture was transformed into competent $E$. coli DH5 $\alpha$. The transformants were initially screened on ampicillin-containing LB agar plate and then re-screened by PCR with specific primers and T7pro/T7ter. Plasmids derived from positive colonies confirmed by PCR colonies were sent to the Macrogen, Korea, for sequencing.

\subsection{Expression of Recombinant SCARB2 in E. coli BL21 (DE3)}

The expression of recombinant SCARB2 was conducted as described with some modifications (Seidmoradei et al. 2020). pET-SCARB2 obtained from previous steps was transformed into competent E. coli BL21 (DE3) strain. Positive colonies were inoculated in LB media shaking tubes supplemented with ampicillin and allowed to grow at $37{ }^{\circ} \mathrm{C}$ in $16 \mathrm{~h}$. The cultures were then sub-cultured at $1: 10$ (v/v) and inoculated at $37{ }^{\circ} \mathrm{C}$ until $\mathrm{OD}_{600}$ reached 0.8-1.0.

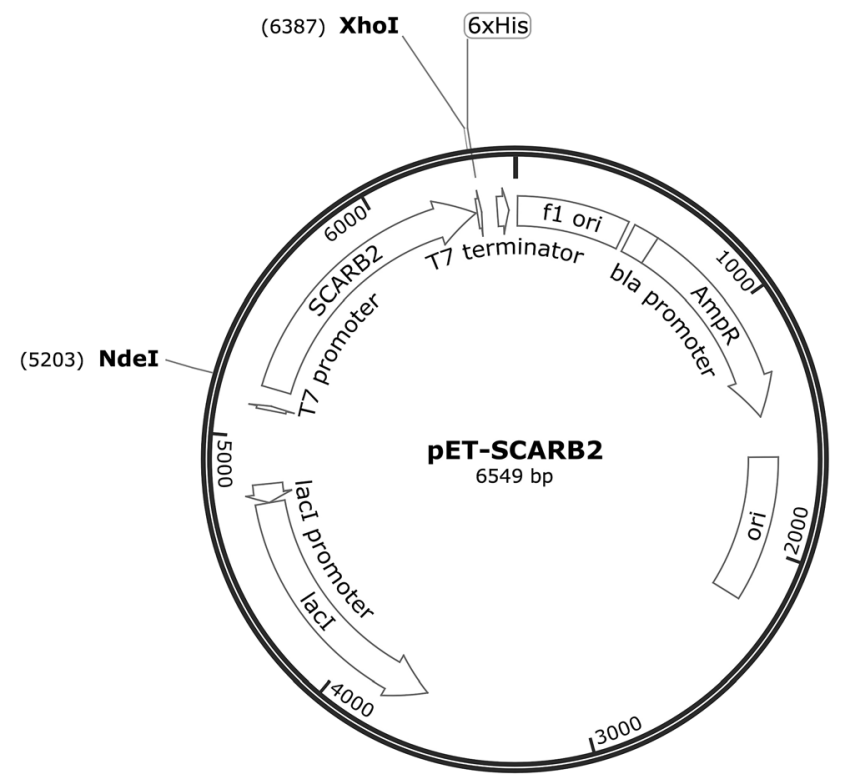

Fig. 1 pET-SCARB2 map 
At this point, the cultures were induced with the final concentration of $0.1 \mathrm{mM}$ IPTG, and the protein expressions were performed at $37{ }^{\circ} \mathrm{C}$ in $4 \mathrm{~h}$. Harvested cells proceeded for sonication on ice in PBS buffer ( $\mathrm{pH}$ 7.4) to obtain proteins in total, soluble and insoluble fractions. A sample of the bacterial culture was taken as negative control with non-induced $E$. coli BL21 (DE3)/pET-SCARB2. Recombinant protein expression was analyzed by SDS-PAGE and Coomassie Brilliant Blue stain, followed by Western blot and probed with His-probe (Santa Cruz) and goat antimouse IgG-HRP antibody (Proteintech). The membrane plot was developed using $100 \mu \mathrm{L}$ of ready-to-use $3,3^{\prime}, 5$, $5^{\prime}$-tetra methyl benzidine (TMB) (Thermo Scientific, 37,574) and incubated at room temperature. The reaction was stopped with PBS buffer and digitized documented.

\subsection{Refolding of SCARB2 Inclusion Bodies}

The refolding of recombinant SCARB2 was conducted as described with some modifications (Tat Truong Dang et al. 2014). Harvested cells proceeded for sonication on ice in lysis buffer (20 mM Tris- $\mathrm{HCl} \mathrm{pH} 8.0,1 \mathrm{M} \mathrm{NaCl}, 5 \mathrm{mM}$ EDTA). The inclusion bodies were obtained by centrifugation at 13,000 rpm for $20 \mathrm{~min}$, then washed five times in washing buffer $(20 \mathrm{mM}$ Tris- $\mathrm{HCl} \mathrm{pH} 8.0,1 \%$ Triton $\mathrm{X}-100$ ) and re-washed in non-Triton washing buffer. Obtained pellet was re-suspended in dissolving buffer (20 mM Tris- $\mathrm{HCl} \mathrm{pH} 8.0,4 \mathrm{M} \mathrm{Gu}-\mathrm{HCl}$ ), well-vortexed and put still for one hour at $4^{\circ} \mathrm{C}$. Supernatant was then harvested using centrifugation at $13,000 \mathrm{rpm}$ for $20 \mathrm{~min}$ and suspended in refolding buffer $(20 \mathrm{mM}$ Tris- $\mathrm{HCl} \mathrm{pH}$ 8.0, 2 M Urea, 10\% Sucrose, $0.1 \%$ Tween 80). Mixture was then well-stirred at $4^{\circ} \mathrm{C}$ for $14 \mathrm{~h}$, and the solubilized cell pellet comprising inclusion bodies was centrifuged at $13,000 \mathrm{rpm}$ for $20 \mathrm{~min}$ to remove insoluble particulates. Finally, the refolding result was verified using SDS-PAGE and silver-stained, followed by Western blot and probed with anti-His (Santa Cruz) and goat anti-mouse IgG-HRP antibody (Proteintech). The membrane plot was developed using $100 \mu \mathrm{L}$ of ready-to-use TMB (Thermo Scientific) and incubated at room temperature. The reaction was stopped with PBS buffer and digitized documented.

The supernatant containing soluble SCARB2 obtained from previous steps was dialyzed against PBS buffer $(\mathrm{pH}$ 7.4) to remove refolding buffer, which could interfere with later experiments. The dialyzed SCARB2 protein was concentrated by 10 MWCO Amicon $\AA$ Ultra centrifugal filter (Thermo Fisher). The obtained SCARB2 concentration was determined by using Bradford assay (Sigma).

\subsection{Enzyme Immunoassay}

The EV71 concentration was determined using TCID50 as previously described (Quynh-Anh Nguyen-Ngoc 2015; Thao et al. 2010). For UV inactivation, the conditions were followed as described (Zou et al. 2013). Briefly, EV71 stock was spread on a Petri dish and then treated with UV irradiation in a Biosafety Cabinet for $30 \mathrm{~min}$ or longer at room temperature. Interaction of recombinant SCARB2 protein with EV71 was evaluated by the indirect enzymelinked immunosorbent assay (ELISA) conducted as described with some modifications (Ding et al. 2015). The 96-well microtiter plates were coated with $10^{5} \mathrm{UV}$-inactivated EV71 virions in $100 \mu \mathrm{L}$ carbonate buffer ( $\mathrm{pH}$ 9.6) at $4^{\circ} \mathrm{C}$ overnight and then blocked with $100 \mu \mathrm{L}$ of $3 \%$ skim milk in PBS-T for an hour at room temperature. After being washed three times with PBS-T, $100 \mu \mathrm{L}$ of recombinant SCARB2 with concentration at $10^{9}, 10^{10}, 10^{11}$ molecules (diluted in PBS pH 7.4 and PBS pH 5.5) was added and incubated at $37^{\circ} \mathrm{C}$ for $2 \mathrm{~h}$. Solutions were removed, and $100 \mu \mathrm{L} /$ well of anti-His-tag-HRP antibody (Proteintech) at 1/20,000 dilution was added and incubated for $1 \mathrm{~h}$ at room temperature. The plate was developed using $100 \mu \mathrm{L}$ of TMB (Sigma, T0440) and incubated at room temperature. The reaction was stopped with $100 \mu \mathrm{L}$ of $\mathrm{HCl}$ $2 \mathrm{~N}$ after $30 \mathrm{~min}$, and the absorbance at $450 \mathrm{~nm}$ was measured on a microplate reader (Thermo). Negative controls were created using no virions coating or no SCARB2 incubating. The cutoff value was calculated as negative control value plus 3 times of SD. The values higher than cutoff value were indicated as positive results.

\section{Results and Discussion}

\subsection{Amplification of scarb2 Gene and Verification of Recombinant pET- SCARB2}

The scarb2 gene was amplified from pPHAGE-C-TAPSCARB2 plasmid using specific primers. The length of the amplified product with about $1179 \mathrm{bp}$ correlated with scarb2 gene (Fig. 2a). Under the PCR conditions performed in our experiments, the scarb2 gene was successfully isolated, which was then cloned into the pET expression vector. PCR colonies were performed to screen for recombinant pET22b-SCARB2 from transformed E. coli $\mathrm{DH} 5 \alpha$. Amplicons with sizes of approximately $1200 \mathrm{bp}$ were obtained by using reversed primer of scarb2 and T7pro (Fig. 2b). The results showed that scarb2 gene was inserted with correct orientation. Sequencing results confirmed the amplified product was in $100 \%$ homologous 
Fig. 2 Amplification of scarb2 gene $\mathbf{a}$ and verification of recombinant plasmid by PCR colonies of E. coli DH5al pET22b-SCARB2 b in $1.5 \%$ agarose gel electrophoresis. M, DNA marker $1 \mathrm{~kb} ; 1$, negative control; 2, amplified scarb2 gene by specific primers; $3-7$, screened colonies by scarb2R primer and $\mathrm{T} 7 \mathrm{pro}$ (a)

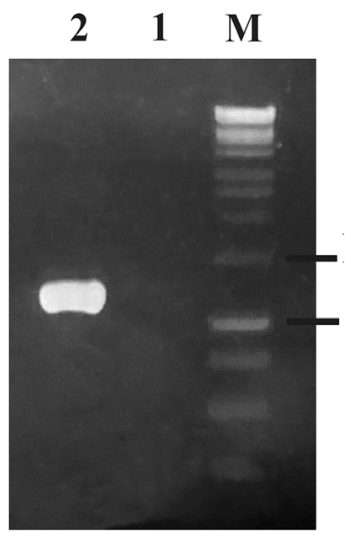

(b)

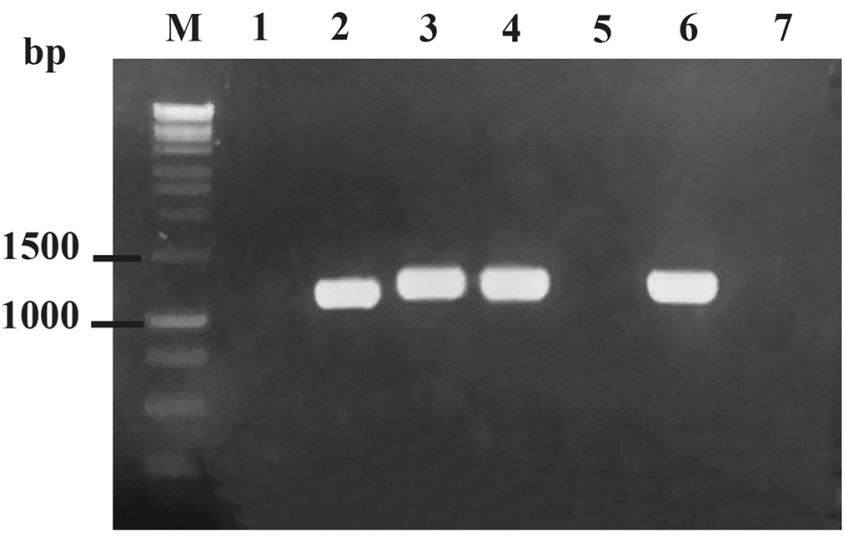

with SCARB2 gene from GenBank (data not shown). Collectively, the recombinant vector of desire was successfully constructed.

\subsection{Expression of Recombinant SCARB2}

The pET22b-SCARB2 vector was transformed into $E$. coli BL21 (DE3) cells. Positive clones were induced by IPTG to produce recombinant proteins. To verify the recombinant SCARB2 proteins, the $E$. coli cells were lysed, subjected to $12.5 \%$ SDS-PAGE and stained with Coomassie Blue. The separated bands on gel showed overexpression of one band at about $45 \mathrm{kDa}$ (lane 2, 3; Fig. 3a), which were exact the predicted sizes of SCARB2. There was no overexpression band in the negative control (lane 4, Fig. 3a). In addition, the SCARB2 was designed to fuse with His-tag at the C-terminal, so the presence of SCARB2 was confirmed using His-probe and goat anti-mouse IgGHRP antibody in Western blot. The results indicated that the protein excessively expressed in the SDS-PAGE gels was SCARB2 (lane 2, 3; Fig. 3b), and this protein expressed mainly in the insoluble fraction. Thus, recombinant SCARB2 fused to His-tag was successfully expressed in E. coli BL21 (DE3) in inclusion bodies.

Human SCARB2 has a theoretical molecular weight of $54.3 \mathrm{kDa}$ (COPaKB 2015). The weight discrepancy between its theoretical $(54.3 \mathrm{kDa})$ and obtained in this study $(45 \mathrm{kDa})$ was due to the deletion of transmembrane domain, which anchors the receptor onto mammalian cell membrane. The extracellular domain was chosen to express since the function of capturing or interacting with viruses solely related to this region. This truncated transmembrane protein could help enhance the solubilization of expressed protein. It could also reduce the chance of transmembrane domain, which contains mostly hydrophobic amino acids, to interact with nascent polypeptides, thereby increasing the solubility of target protein. SCARB2's structure has been confirmed as it carries 10 high mannose-type $\mathrm{N}$-linked oligosaccharide chains and two disulfide bridges, which contribute to the receptor in its proper folding (Dang et al. 2014; Yamayoshi and Koike 2011). Glycosylation and disulfide bridges could have facilitated the ability of
Fig. 3 Coomassie brilliant blue staining of expressed SCARB2 analyzed by SDS-PAGE on $12.5 \%$ gel a and confirmed by Western blot probed with antiHis, b M, protein maker a and pre-stained protein marker $\mathbf{b}$; 1-3, E. coli BL21 (DE3)/ pET22b-SCARB2 (+ IPTG); 1, soluble phase; 2 , insoluble phase; 3 , total phase; 4, E. coli BL21 (DE3)/pET22b-SCARB2 (-IPTG) (a)

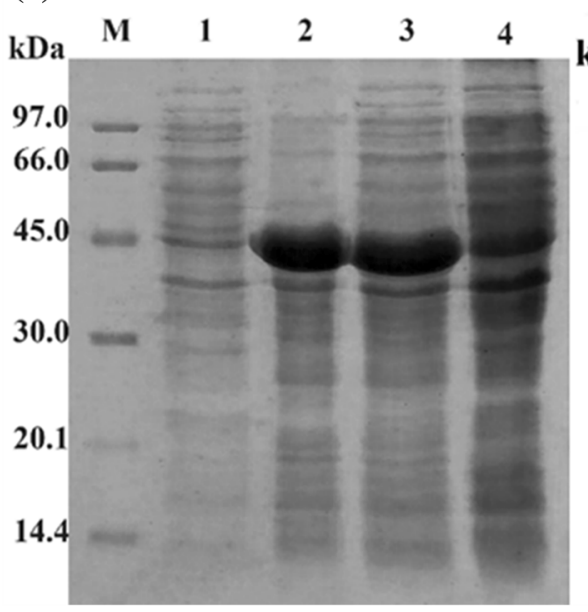

(b)

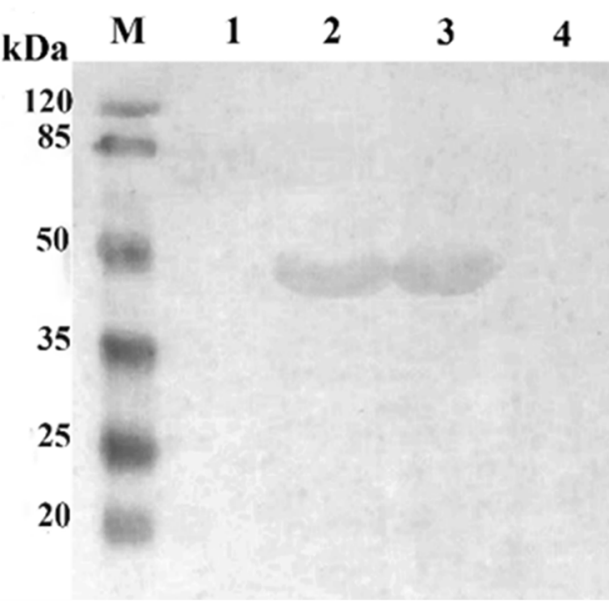


interacting with virus capsid. Therefore, elimination of these factors to study the dependence of receptor function was performed. As E. coli expression system does not facilitate post-translation modifications like glycosylation, which has resulted in accumulation of recombinant product in the form of insoluble inclusion bodies, this obstacle might lead to difficulties in further experiments; therefore, a refolding procedure was necessary.

\subsection{Solubilization and Refolding of Recombinant SCARB2 from Inclusion Bodies}

SCARB2 obtained from E. coli BL21 (DE3)/pET22bSCARB2 formed inclusion bodies. The suspension of inclusion bodies in the washing buffer containing $1 \%$ Triton X-100 aided for the inclusion bodies cleaning process. Dissolving in high molarity urea, $\mathrm{Gu}-\mathrm{HCl}$ containing buffer, $10 \%$ sucrose and $0.1 \%$ tween 80 contributed to complete denaturation and refolding of targeted protein. After extraction from the inclusion bodies with a refolding buffer, the final product was dialyzed and concentrated by 10 MWCO. Finally eliminating the molarity of urea, Gu$\mathrm{HCl}$ concentration by dialysis contributed to increase proper folded protein, as well as ensured the ability of SCARB2 in later experiments.

As can be observed from the electrophoresis gel, the targeted protein bands in lane 1, lane 3 and lane 5 were at corresponding molecular weight (Fig. 4a). Simultaneously, the targeted bands that appeared in lane 2 indicated that the dissolution step was not fully completed. However, the targeted bands disappeared in lane 4 , which showed that the protein of interest was successfully refolded. The refolding efficiency was about $100 \%$. As fused with Histag at the C-terminal, the refolding result was confirmed by Western blot using anti-His (Fig. 4b). The corresponding bands on the membrane plot proved the success of our refolding method. This was the first report of obtaining the soluble form of SCARB2 from inclusion bodies (Xu et al. 2018).

\subsection{Evaluation of the Interaction between Recombinant SCARB2 and EV71}

Indirect ELISA was conducted to determine the interaction between recombinant SCARB2 and EV71 at two different $\mathrm{pHs}$ (5.5 and 7.4). In order to investigate the ratio of interaction between EV17 and SCARB2, molecule was utilized instead of concentration. As revealed in the previous study, a conformational change was observed in SCARB2 induced by $\mathrm{pH}$, which led to a noticeable dissimilarity in a specific region between structures at two $\mathrm{pH}$ levels as an implication for the receptor's function (Dang et al. 2014). Our result showed that recombinant SCARB2 and EV71 did have interaction in dose-dependent manner at both $\mathrm{pH}$ levels, but at lower $\mathrm{pH}$ SCARB2 (at $10^{11}$ dilution) exhibited a significantly higher interaction, which was approximately 5 times higher compared to that of $\mathrm{pH}$ 7.4 (Fig. 5). Firstly, this result was in line with Yamayoshi et al. (2011), supporting the finding that SCARB2 was an EV71 main receptor. Secondly, this result was obtained under soluble form of SCARB2, which was different from $\mathrm{Xu}$ et al. (2018) as their insoluble protein product. Moreover, critical region of SCARB2 accountable for binding to EV71 was identified (Chen et al. 2012; Yamayoshi and Koike 2011). Zhao et al. (2014) stated that pH 5.5 was the point at which the binding region showed significant flexibility, whereas at higher $\mathrm{pH}$ level, this region was more compact and steadier. This crucial observation contributed to the explanation of differences between the two $\mathrm{pH}$-induced interactions.

There have been considerations about the role of glycosylation in SCARB2. Yamayoshi et al. (2011) stated that the N-linked carbohydrate chains of human SCARB2 were not necessary for the EV71-SCARB2 interaction. Nevertheless, the study of Dang et al. (2014) indicated that the
Fig. 4 Silver staining of dissolution and refolding results of recombinant SCARB2 by SDS-PAGE on $12.5 \%$ gel a and validated by Western blot probed with anti-His b. M, protein maker $\mathbf{a}$ and pre-stained protein marker $\mathbf{b} ; 1$, inclusion bodies; 2, precipitated phase after dissolving; 3 , supernatant phase after dissolving; 4 , precipitated phase after refolding; 5, supernatant phase after refolding (a)

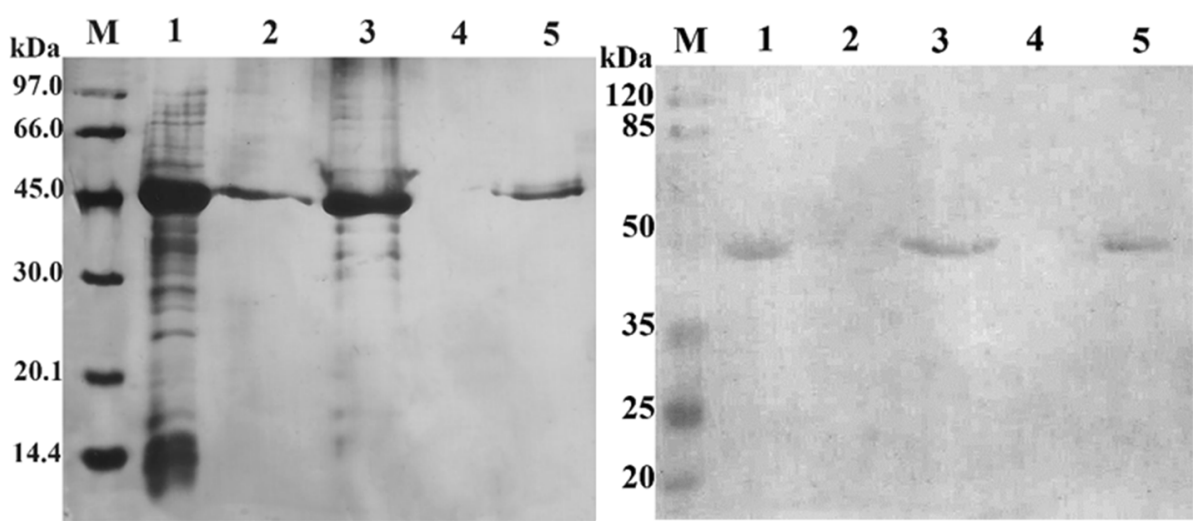



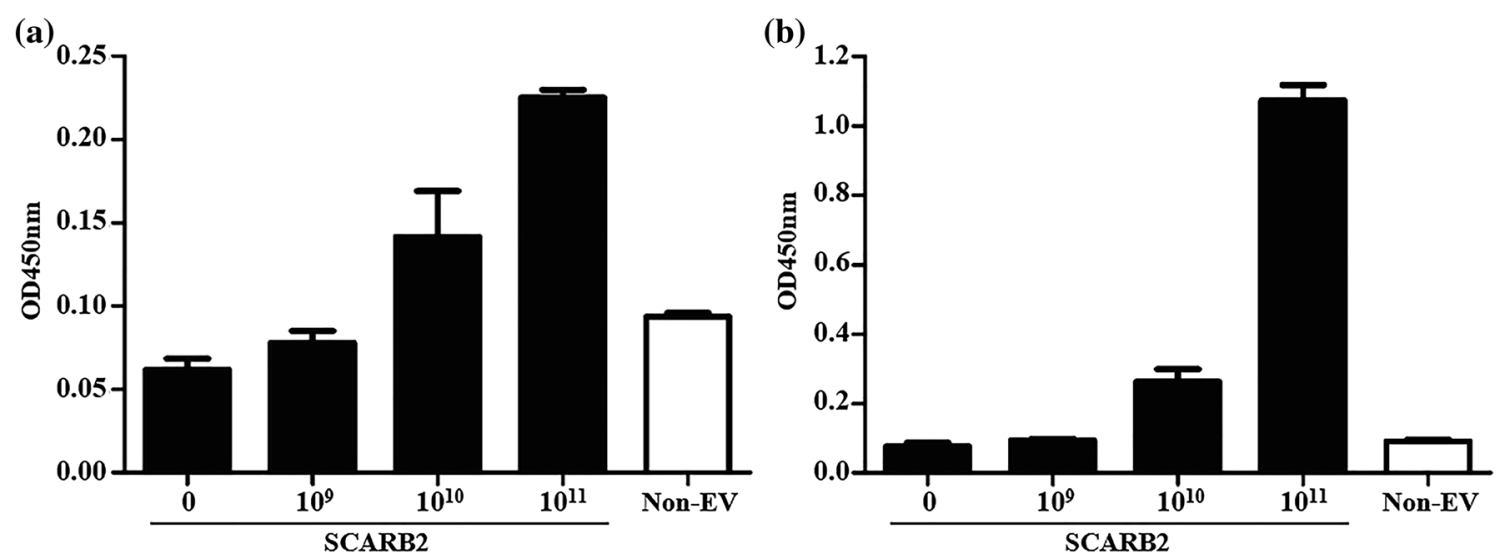

Fig. 5 The interaction of recombinant SCARB2 and EV71 determined by indirect ELISA at pH 7.4 a and pH 5.5 b

attachment of SCARB2 to EV71 decreased when glycosylation was reduced. In agreement with this opinion, Zhao et al. (2014) confirmed the role of glycan as an attachment point for interaction between SCARB2 and EV71 due to the finding of partial cover of glycan to the residues responsible for viral attachment region. The discrepancy was partly revealed in this study. Our soluble and nondisulfide, non-glycosylated receptor had showed the ability to attach with EV71 in vitro, despite low signals. Upon acidification, the binding signals increased. It could be that SCARB2 underwent confirmation changes, thereby exposing viral attachment region though we could not exclude the role of post-translation modifications. This could indicate that conformational change played a more important role in virus binding than post-translation modifications. Therefore, further experiments need to be conducted for fully understanding the principal mechanism. On the other hand, SCARB2-soluble form could facilitate the initial step for cell and in vivo experiments, as the expression system using E. coli BL21 (DE3) could not provide needed post-translation modifications like glycosylation and disulfide bridges, which might affect the attachment efficacy. Consequently, to clarify the unclear role of glycosylation, higher expression systems should be employed. The E. coli Origami strain and yeast expression system could be potential candidates for further investigations.

\section{Conclusion}

In this study, the recombinant vector carrying the gene scarb2 (pET22b-SCARB2) encoding for SCARB2 protein was successfully constructed. Transformed E. coli BL21 (DE3) with recombinant vector pET22b-SCARB2 was successfully created. Extraction of SCARB2 from inclusion bodies was first time documented with a yield of
$100 \%$. Interaction of EV71 and recombinant SCARB2 was confirmed via indirect ELISA. To our knowledge, our present study documented for the first time a procedure for obtaining soluble form of E. coli-derived SCARB2 using refolding method. Because early steps of viral infection require crucial interactions between host cell receptors and viruses, blocking or interfering with the interactions could have potential applications. Due to its wide distribution in human cells and organs (Kuronita et al. 2002), entrance through one of the main EV71's receptors SCARB2 could bring more severe consequences to patients. On that account, appropriate strategies, such as receptor-displaying probiotics or generally recognized as safe organisms, for utilizing recombinant SCARB2 should be performed to help reduce the harm of EV71 on children during the epidemic seasons.

Author contributions All authors contributed to the study conception and design. All authors read and approved the final manuscript.

Funding Hai-Vy Vo-Nguyen was funded by Vingroup Joint Stock Company and supported by the Domestic Master/PhD Scholarship Programme of Vingroup Innovation Foundation (VINIF), Vingroup Big Data Institute (VINBIGDATA).

\section{Compliance with ethical standards}

Conflicts of interest The authors declare that they have no conflict of interest.

\section{References}

Brown BA, Pallansch MA (1995) Complete nucleotide sequence of enterovirus 71 is distinct from poliovirus. Virus Res 39:195-205

Chen P et al (2012) Molecular determinants of enterovirus 71 viral entry cleft around Gln-172 on VP1 protein interacts with variable region on scavenge receptor B 2. J Biol Chem 287:6406-6420 
COPaKB (2015) Protein sequence of human SCARB2 (Uniprot ID: Q14108).

Dang M et al (2014) Molecular mechanism of SCARB2-mediated attachment and uncoating of EV71. Protein cell 5:692-703

Ding Y et al (2015) Characterization of the antibody response against EV71 capsid proteins in Chinese individuals by NEIBM-ELISA. Scientific reports 5:1-11

Huttlin EL et al (2015) The BioPlex network: a systematic exploration of the human interactome. Cell 162:425-440

King A et al (2000) Virus taxonomy: Seventh report of the International Committee for the Taxonomy of Viruses. Picornaviridae. Academic Press, New York and San Diego, CA, pp 657-673

Kuronita T, Eskelinen E-L, Fujita H, Saftig P, Himeno M, Tanaka Y (2002) A role for the lysosomal membrane protein LGP85 in the biogenesis and maintenance of endosomal and lysosomal morphology. J cell sci 115:4117-4131

Lee KY (2016) Enterovirus 71 infection and neurological complications Korean. J pediatrics 59:395

Li $\mathrm{R}$ et al (2014) An inactivated enterovirus 71 vaccine in healthy children New England. J Med 370:829-837

Quynh-Anh Nguyen-Ngoc T-TTN, Dang-Trinh M-A, Van Nguyen K, Tran B-P, Nguyen H-L, Le-Phan K-N, Le-Ha T-D, Hoang-Ngoc K-Q, Le LT, Nguyen Y-N, Nguyen T-T, Cao B-V (2015) Enterovirus 71 (EV71) neutralization effect of $\operatorname{IgY}$ antibodies obtained from egg yolk Vietnam. Journal of Preventive Medicine 5:144

Seidmoradei R, Zeinoddini M, Saeedinia AR, Xhodadadi N (2020) Intein-mediated fusion soluble expression of recombinant human interferon beta (rhIFN- $\beta$ ) Iranian. J Sci Technol Trans A: Sci 44:371-377

Dang TT, Trinh MT, Tran-Van H (2014) Cloning, expression and purification of human fibroblast growth factor 1 (FGF-1) in Escherichia coli. J Biotechnol 12:615-621

Thao NTT, Ngoc NTK, Tú PV, Thúy TT, Cardosa MJ, McMinn PC, Phuektes P (2010) Development of a multiplex polymerase chain reaction assay for simultaneous identification of human enterovirus 71 and coxsackievirus A16. J virol methods 170:134-139
WHO The WHO Regional Office for the Western Pacific's Institutional Repository for Information Sharing. https://iris.wpro.who. int/.

WHO (2011) A guide to clinical management and public health response for hand, foot and mouth disease (HFMD). WHO Regional Office for the Western Pacific, Manila

Xu T, Lin Z, Wang C, Li Y, Zhao M, Hua L, Zhu B (2018) Prokaryotic expression and identification of scavenger receptor B2. Acta Virol 62:50-57

Yamayoshi S, Fujii K, Koike S (2012) Scavenger receptor B2 as a receptor for hand, foot, and mouth disease and severe neurological diseases. Frontiers in microbiology 3:1-6

Yamayoshi S et al (2012) Human SCARB2-dependent infection by coxsackievirus A7, A14, and A16 and enterovirus 71. J virol 86:5686-5696

Yamayoshi S, Koike S (2011) Identification of a human SCARB2 region that is important for enterovirus 71 binding and infection. J virol 85:4937-4946

Yamayoshi S, Yamashita Y, Li J, Hanagata N, Minowa T, Takemura T, Koike S (2009) Scavenger receptor B2 is a cellular receptor for enterovirus 71. Nat med 15:798-801

Yi E-J, Shin Y-J, Kim J-H, Kim T-G, Chang S-Y (2017) Enterovirus 71 infection and vaccines. Clin exp vaccine res 6:4-14

Zhu F-C et al (2013) Efficacy, safety, and immunology of an inactivated alum-adjuvant enterovirus 71 vaccine in children in China: a multicentre, randomised, double-blind, placebo-controlled, phase 3 trial. The Lancet 381:2024-2032

Zhu $F$ et al (2014) Efficacy, safety, and immunogenicity of an enterovirus 71 vaccine in China New England. J Med 370:818-828

Zou S et al (2013) Inactivation of the novel avian influenza A (H7N9) virus under physical conditions or chemical agents treatment. Virol J 10:289

Zhao Y, Ren J, Padilla-Parra S, Fry EE, Stuart DI (2014) Lysosome sorting of $\beta$-glucocerebrosidase by LIMP-2 is targeted by the mannose 6-phosphate receptor. Nature Commun 5(1):4321 